\title{
2005
}

\section{Physics and Advanced Technologies}

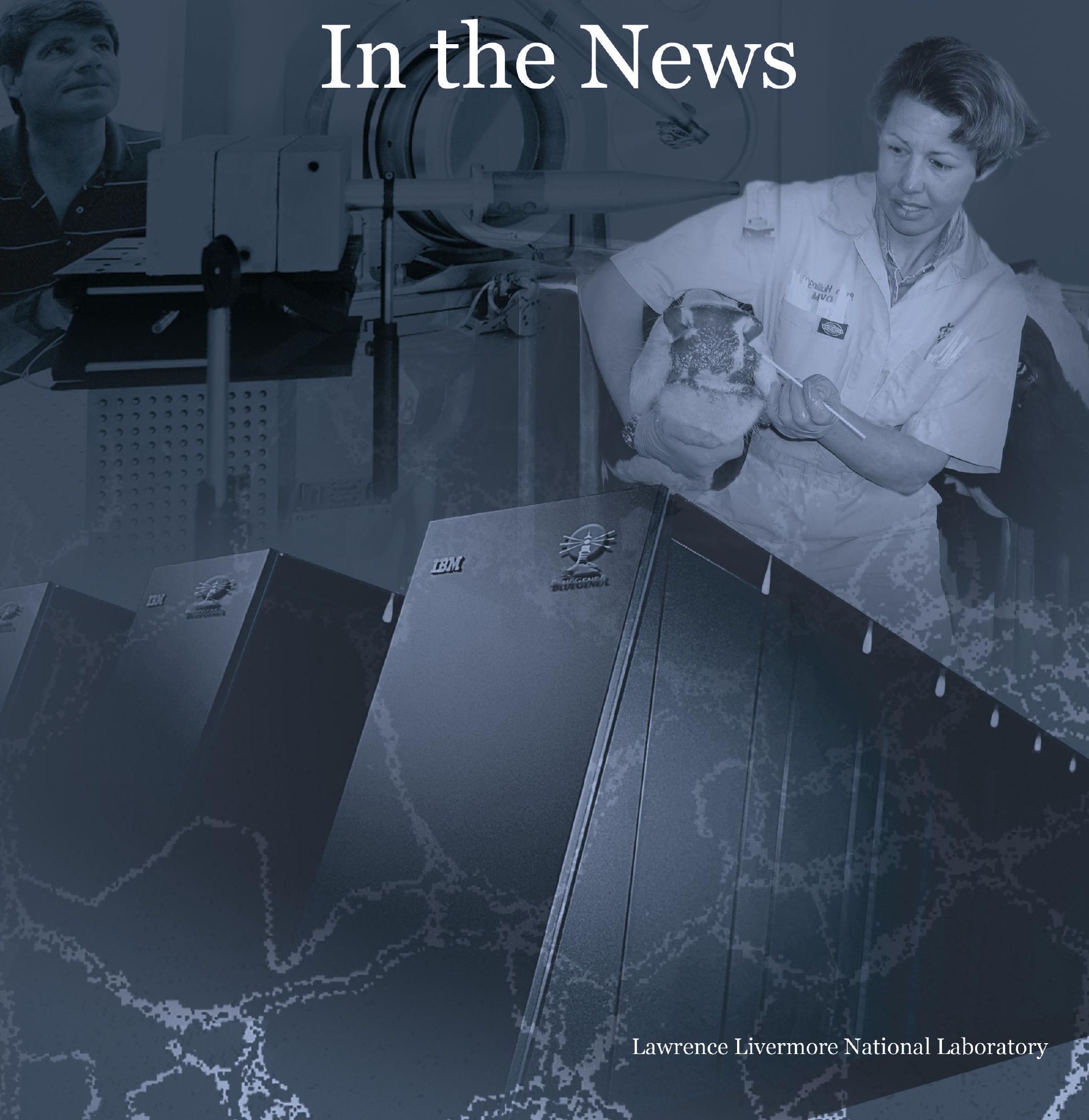




\section{5}

Physics and Advanced Technologies

In the News

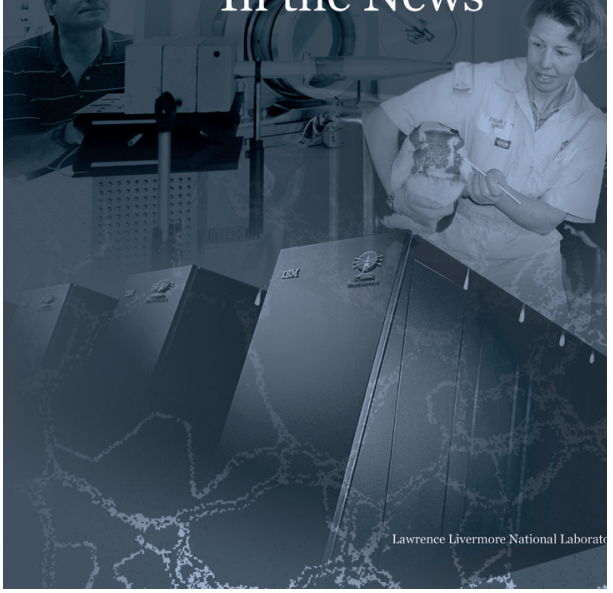

Editor

Andrew Hazi

Designer

Amy Henke

Proofreader

Maurina Sherman

\section{About the Cover}

Physicist Dwight Price examining the inside of the target chamber of the recently commissioned Titan laser. Titan is one of the few petawatt-class lasers in the world and is part of the Jupiter Laser Facility operated by Physics and Advanced Technologies (PAT) at Livermore (see p. 7).

Veterinarian Pam Hullinger, from the Medical Physics and Biophysics Division in PAT, collecting samples at the animal science facilities of the University of California at Davis. The samples were used to test new diagnostics developed at Livermore for the rapid detection of the foot-and-mouth disease virus (see p. 11).

Part of BlueGene/L, currently the world's fastest high performance computer. Superimposed is an image of the grain boundaries resulting from a 16-million-atom simulation of the transition from molten to solid tantalum. A Livermore-IBM team received the 2005 Gordon Bell Prize for this path-breaking calculation using BlueGene/L (see pp. 10 and 16).

\section{About this Report}

Several outstanding research activities in the Physics and Advanced Technologies Directorate in 2005 were featured in Science and Technology Review, the monthly publication of Lawrence Livermore National Laboratory. Reprints of those articles accompany this report. Here we summarize other science and technology highlights, as well as the awards and recognition received by members of the Directorate in 2005. As part of the World Year of Physics commemorating the 100th anniversary of Einstein's "miraculous year," we also highlight ongoing physics research that would not be possible without Einstein's pioneering accomplishments.

\section{About Physics and Advanced Technologies}

The Physics and Advanced Technologies Directorate was established in July 2000 through the merger of the former Physics Directorate and elements of the former Laser Programs. The Directorate has a budget of approximately $\$ 160$ million and a staff of approximately 380 employees. We are highly integrated and multidisciplinary, with substantive collaborations with the rest of Lawrence Livermore National Laboratory and with other national laboratories, universities, and industries. Our mission is to be a leader in frontier physics and technology for 21 st-century national security missions: stockpile stewardship, homeland security, global stability, and scientific preeminence.

\section{Find Out More About Us}

Visit our Web site at http://www-pat.IInl.gov/ for more information on Physics and Advanced Technologies research, facilities, publications, staff, organization, events, and awards.

This document was prepared as an account of work sponsored by an agency of the United States Government. Neither the United States Government nor the University of California nor any of their employees, makes any warranty, express or implied, or assumes any legal liability or responsibility for the accuracy, completeness, or usefulness of any information, apparatus, product, or process disclosed, or represents that its use would not infringe privately owned rights. Reference herein to any specific commercial product, process, or service by trade name, trademark, manufacturer, or otherwise, does not necessarily constitute or imply its endorsement, recommendation, or favoring by the United States Government or the University of California. The views and opinions of authors expressed herein do not necessarily state or reflect those of the United States Government or the University of California, and shall not be used for advertising or product endorsement purposes.

This work was performed under the auspices of the U.S. Department of Energy by University of California, Lawrence Livermore National Laboratory under Contract W-7405-Eng-48.

UCRL-TR-227148
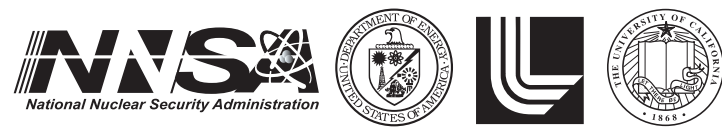


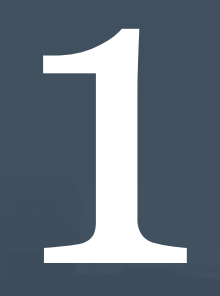

\section{Research in the News}

Advancing science and technology

in the national interest 
Analysis of interplanetary dust yields clues

Laboratory researchers have detected and identified a 2,175-angstrom feature (or bump) in the absorption spectra associated with interstellar grains embedded within interplanetary dust particles (IDPs). Using a transmission electron microscope, they identified organic carbon and amorphous silicarich material as the source of the 2,175-angstrom spectral feature. This discovery may help explain how some IDPs formed from materials found in interstellar space.

The team's results, which were published in the January 14, 2005, issue of Science, suggest that the carbon and silicate grains may have been produced by irradiation of dust in the interstellar medium. The measurements may help explain how interstellar organic matter was incorporated into the solar system. "Our finding potentially breaks a logjam in the search for the carrier of the astronomical 2,175-angstrom bump," says John Bradley, director of Livermore's Institute of Geophysics and Planetary Physics (IGPP) and lead author of the paper. "Over the past 40 years, a variety of exotic materials have been proposed. Our findings suggest that organic carbonaceous matter and silicates, the 'common stuff' of interstellar space, may be responsible for the 2,175-angstrom bump."

The Livermore work was funded by the Laboratory Directed Research and Development (LDRD) Program. Other collaborators on the project include researchers from the University of California at Davis, Lawrence Berkeley National Laboratory, Washington University, and the National Aeronautics and Space Administration's Ames Research Center.

Contact: John Bradley (925) 423-0666 bradley33@IInl.gov
Telescope to peer into deep sky

On January 26, 2005, the National Aeronautics and Space Administration (NASA) announced approval of a collaborative study to build and launch the Nuclear Spectroscopic Telescope Array (NuSTAR). Led by the California Institute of Technology, the collaboration also includes researchers from Lawrence Livermore, Columbia University, the Danish

Space Research Institute, NASA's Jet Propulsion Laboratory, and several other institutions.

Laboratory scientists will use the pioneering telescope to understand how stars explode and produce elements such as calcium. With NuSTAR, scientists will for the first time be able to obtain a high-energy (hard) $\mathrm{x}$-ray map of the sky in extraordinary resolution. The telescope can be used to study supernovae phenomena and the accretion of matter by black holes. NuSTAR technologies may also have applications in homeland security because hard $x$ rays are a key method for detecting and identifying nuclear material.

Telescopes have taken images of the deep sky in the optical, infrared, and low-energy (soft) x-ray bands. However, most black holes are obscured by dust and cannot be seen in images of those bands. Hard $\mathrm{x}$-ray telescopes can penetrate this dust to detect even the most obscured supermassive black holes. NuSTAR will be hundreds of times more sensitive than any previous hard $x$-ray instrument, which will greatly improve the image resolution.

Scientists are particularly interested in the information hard $x$-ray imaging will provide on the deepest layers of exploding stars and the new chemical elements they produce. For example, titanium-44 is an interesting tracer element for studying what happened in a supernova. Also, when titanium-44 
decays, it is dispersed throughout the universe in the form of calcium, which eventually winds up in human bones and teeth.

NASA will review the project in early 2006 to make a decision on proceeding to flight development for launch.

\section{Contact: Bill Craig (925) 423-1471 billc@llnl.gov}

Reprinted from May 2005 issue of Science \& Technology Review of the Lawrence Livermore National Laboratory.

\section{Advanced detectors reduce the threat of nuclear terrorism}

In January 2005, Discover magazine named its "top 100 stories," including a new radiation detection technology being developed by the Laboratory to counter the threat of nuclear or radiological terrorism. The device can detect highly enriched uranium or plutonium inside cargo containers through active interrogation of the contents using high-energy neutrons. Following neutron irradiation, the time-delayed, high-energy gammaray emission from nuclei produced by beta decay provides a unique diagnostic signature.

In early experiments using a 14-MeV neutron source, the researchers observed large background signals due to gamma rays from ${ }^{16} \mathrm{~N}$ produced by neutron activation of oxygen that is present in agricultural products or plastics. In 2005, the team installed a new neutron source capable of producing neutrons with energies in the 1-3 and 3-7 million electron volt range. They also developed surrogate cargo samples, such as stacks of plywood to simulate agricultural or fuel cargo, stacked aluminum sheets to represent electronics cargo, and stacked steel pipes to simulate heavy machinery. Experiments conducted with lower-energy neutrons on highly enriched uranium covered with solid wood or steel pipes up to four feet thick, produced gamma-ray signals with good sensitivity for even a single neutron irradiation. When 3-7 million electron volt neutrons were used, the signals due to the highly enriched uranium were six standard deviations above the background, demonstrating a breakthrough in eliminating the interference produced by neutron activation of oxygen. The researchers are now testing a deuterium gas target in the neutron source, which produces a higher yield of neutrons in the 3-7 million electron volt energy range, better suited for interrogating thick cargos. Experiments are also underway to address issues that could produce false positives due to variations in background signals as the cargo moves about or as different cargo materials pass in front of the detector.

\section{Contact: Dennis Slaughter (925) 422-6425 slaughter1@IInI.gov}

\section{Researchers study the transformation of neutrinos}

As part of the international team working on the Main Injector Neutrino Oscillation Search (MINOS) experiment, Livermore scientists hope to study, in detail, the transformation of neutrinos from one type to another. Neutrinos are relatively massless particles with no electric charge, but they are fundamental to the makeup of the universe. To explore the mysterious nature and properties of neutrinos, the MINOS researchers will use two giant detectors - one at Fermi National Accelerator Laboratory (Fermilab) and a 5,400-ton detector lying in a historic iron mine at Soudan, Minnesota.

One goal of the MINOS experiment is to discover the rate at which neutrinos change "flavors," or oscillate from one type to another. Neutrinos are difficult to detect because they rarely interact with anything. Although they 
can easily pass through a planet or solid walls, they seldom leave a trace of their existence.

In the MINOS experiment, a narrow beam of neutrinos is generated and characterized by the near detector at Fermilab. The beam is aimed at a far detector, located in the Soudan Underground Laboratory in Minnesota. The neutrino beam energy is chosen so that the distance between the two detectors corresponds to an expected maximum in the probability that a neutrino produced at Fermilab will oscillate to another flavor. Studying the elusive neutrino will help scientists better understand particle physics, specifically the role of neutrinos in the formation of the universe and their relationship to dark matter.

Dedication ceremonies for the MINOS detectors were held at Fermilab on March 4, 2005. Livermore's MINOS research is funded by the Laboratory Directed Research and Development and Physical Data Research programs. The MINOS experiment as a whole is funded by the Department of Energy's Office of Science.

Contact: Peter Barnes (925) 422-3384 pdbarnes@lInl.gov

Reprinted from May 2005 issue of Science \& Technology Review of the Lawrence Livermore National Laboratory.

\section{Measuring the composition of} Titan's atmosphere

Livermore is collaborating with the National Aeronautics and Space Administration (NASA), the University of British Columbia (UBC), and NASA's Jet Propulsion Laboratory (JPL) to measure the temperature, winds, and chemical composition of Titan's atmosphere. Titan is one of Saturn's moons. Led by principal investigator F. Michael Flasar of
NASA's Goddard Space Flight Center, the team is using the Cassini Composite Infrared Spectrometer (CIRS) on the Cassini-Huygens spacecraft for these measurements.

CIRS measures the intensity of far-infrared radiation, light with wavelengths between those of radar and near-infrared light. The abundance of methane in Titan's atmosphere is determined by comparing the intensity of spectral emission lines from the atmosphere with laboratory measurements. Livermore physicist Edward Wishnow measured the spectrum of methane at temperatures and densities similar to Titan's - about 113 kelvins and about 1 atmosphere of pressure. Because the laboratory absorption spectra correspond well with the Titan spectral lines, the laboratory results can be used to determine the amount of methane in Titan's upper atmosphere. UBC scientists Herbert Gush, Irving Ozier, and Mark Halpern collaborated on the laboratory work, and JPL scientist Glenn Orton helped interpret the data.

Titan is the only moon in the solar system with a substantial atmosphere, and that atmosphere is primarily composed of nitrogen, making it similar to Earth's. Scientists want to study Titan's atmosphere because the organic chemistry occurring there is analogous to the processes that may have occurred in the early terrestrial atmosphere. CIRS observations of Titan's stratosphere also indicate that its winter (northern) pole has many properties in common with Earth's. Titan's cold temperatures, strong circumpolar winds, and concentrations of several compounds are analogs to the polar winds and ozone hole on Earth. Both also have strong winds that isolate the polar air and inhibit mixing with air at lower latitudes. 
Cassini-Huygens is an international collaboration between NASA and the European and Italian space agencies. Cassini is the first spacecraft to explore the Saturn system of rings and moons from orbit. Results from the team's research appeared in the May 13, 2005, issue of Science.

Contact: Edward H. Wishnow (925) 423-1471 wishnow@IInl.gov

Reprinted from July/August 2005 issue of Science \& Technology Review of the Lawrence Livermore National Laboratory.

\section{Electron cloud effects in heavy-ion beams}

A team of researchers from Lawrence Livermore and Lawrence Berkeley National Laboratory (LBNL) is advancing the science underpinning the performance of high-intensity accelerators for heavy-ion inertial fusion, high energy density physics, and highenergy physics. In measurements done at the High-Current Experiment at LBNL, the team characterized the stray electron clouds and beam-induced gas desorption that result from ions in the beam hitting metal surfaces (e.g., beam tube) at grazing angles. These effects are known to limit the performance of high-current accelerators.

The researchers measured an electron emission coefficient of 100 and a gas desorption coefficient of 10,000 for million-electron-volt potassium ions bombarding stainless steel. lon scattering from the surface exacerbates the emission and desorption by allowing ions to impact the surface multiple times. It also causes the ions to hit the wall in regions where the magnetic field allows the stray electrons to fill the entire volume.

The variation of the emission coefficient observed with changing the angle of incidence of the impacting ions was consistent with electron emission originating from a very thin (2-nanometer) surface layer. The angular dependence of electron emission and ion scattering suggested an approach to mitigating the effects of beaminduced electron emission and gas desorption. By artificially roughening the metal surface (at 100-micrometer scale) by fine sand blasting, the researchers were able to increase the probability that ions impacted at angles closer to normal incidence (near the rim of the craters). Using the roughened surface, the electron emission coefficient was reduced below 15, and the gas desorption coefficient to less than 4,500.

The team has also demonstrated an in-situ fast-ionization gauge that measures gas density within an ion beam. This diagnostic is applicable to space-charge dominated beams with relatively high fill factor, where cold ions can be expelled across the quadrupole magnetic field by the beam potential. This diagnostic also provides a measurement of the rate of electron generation by beam-impact ionization of the gas. The results of this research were published in the May 21, 2005 issue of Nuclear Instruments and Methods in Physics Research, A. Contact: Art Molvik (925) 422-9817 molvik1@IInl.gov

\section{Training the next generation of scientists in high energy density physics}

Livermore's Institute for Laser Science and Application (ILSA) partnered with the Fusion Science Center at the University of Rochester to host the 2005 High Energy Density Physics (HEDP) Summer School, held 
August 7-13, 2005 at the Clark Kerr Campus of the University of California, Berkeley. As a discipline, HEDP focuses on the collective properties of, and phenomena associated with, matter that is in a state characterized by high energy density $\left(10^{11}\right.$ joules per cubic meter or greater). Fusion plasmas, supernovae, and materials heated by intense ultra-short pulse lasers or relativistic particle beams are just a few examples of high energy density matter. Participants at the Summer School included undergraduate seniors, graduate students, postdoctoral researchers, and early career academic researchers who wanted to explore, or advance their knowledge of, high energy density physics. Lecture topics included radiation transport and spectroscopy, hydrodynamics, materials properties under extreme conditions, laserplasma interactions, and experimental techniques, along with ongoing research activities in HED science. More than 50 scholarships covering meals, lodging, and travel expenses were made available to undergraduate seniors, graduate students, and postdocs who through an application process indicated their interested in attending. Proceedings from the HEDP Summer School are available from the Web site: http://meetings.lle.rochester. edu/FSCschool/proceedings.html. Additional background information about the topics discussed at the Summer School can be found at the Fusion Science Center's HEDP Web site: http://fsc.lle.rochester.edu/hedp. $\mathrm{html}$. Descriptions of other related conferences and workshops sponsored, or co-sponsored, by ILSA are described at the Web site: http://ilsa.IInl.gov/.

Contact: Don Correll (925) 422-6784 correll1@IInl.gov

\section{Scientists reveal the way nanotubes grow}

In work funded by the Laboratory Directed Research and Development (LDRD) Program, Livermore scientists have clarified the growth process for nanotubes - molecules that are typically 10,000 times smaller than the diameter of a human hair. Because nanotubes are extremely strong and have good thermal conductivity, researchers want to use them in nanoscale electronic and mechanical applications. By understanding their growth structure, researchers will be better able to manipulate the use of nanotubes in technological applications.

The Livermore team performed a series of quantum molecular dynamics simulations using different initial conditions for carbon coverage on iron nanoparticles. The team chose iron as a catalyst because experimentally it has the highest rate of carbon nanotube production. In the simulations, carbon atoms bonded to the iron, forming chains that eventually interconnected to form a sheet of pentagons and hexagons.

"We were surprised that carbon and iron do not mix at the nanoscale during growth and that the tubes grew capped," says Giulia Galli, a physicist in Livermore's Physics and Advanced Technologies Directorate. "Our simulations clarified the growth of nanotubes on small metal nanoparticles and thus will help researchers design experiments aimed at controlled growth of nanotubes." The team's research appeared in an article that was highlighted on the cover of the August 26, 2005, issue of Physical Review Letters, and was 
featured in the October 2005 issue of Nature Materials.

Contact: Giulia A. Galli (530) 754-9554 galligygi2@IInl.gov

Reprinted from December 2005 issue of Science \& Technology Review of the Lawrence Livermore National Laboratory.

\section{Experiments to understand plutonium}

Plutonium is an extremely complex material, critically important to the functioning of nuclear weapons. One of the major scientific challenges of the Stockpile Stewardship Program is to understand the detailed properties of plutonium metal and alloys and how they age. Experiments at the Joint Actinide Shock Physics Experimental Research (JASPER) Facility and with diamond anvil cells are providing new data for properties of this material.

Located at the Nevada Test Site, JASPER is a 30-meter-long, two-stage gas gun that accelerates a projectile to speeds of up to 8 kilometers per second. In a JASPER experiment, the impact of the projectile produces an extremely high-pressure shock wave (about 600 gigapascals or 6 million atmospheres) in the targeted material, raising its temperature to as high as 7,000 kelvins. Through precise measurements of shock velocity, scientists are improving the equationof-state models of plutonium used in simulations of weapon performance. Although fewer tests were performed at JASPER than during the previous year, all experiments produced excellent data and several broke new ground. For example, researchers acquired the first data to test the equation of state of aged plutonium. The experiment achieved the expected high accuracy, enabling direct comparison of dynamic compression data for aged and unaged plutonium samples. The researchers also conducted the first quasi-isentropic compression experiment on plutonium using a newly developed projectile with graded density. This projectile is made of materials with smoothly varying density from front to back, designed specifically to produce a steady compression of the target without shocking it. The technique allows scientists to study the response of compressed materials in different thermodynamic regimes than those accessible in shock experiments.

Laboratory experiments using diamond anvil cells complement shock physics experiments. A diamond anvil cell is a small mechanical press that squeezes a microgram of material between two small, flat-tipped diamonds, achieving pressures as high as 100 gigapascals. In diamond anvil experiments fielded at the Advanced Photon Source at Argonne National Laboratory, and at other synchrotron light sources, Livermore scientists obtained high-accuracy equation-of-state data for plutonium samples whose ages ranged from 0 to 45 years. Experiments also characterized differences in equations of state among several plutonium alloys and validated theoretical models of phase transitions.

Contact: Neil Holmes (925) 422-7213 holmes4@IInl.gov on JASPER, or Hyun-chae Cynn (925) 422-3432 cynn1@IInl.gov on DAC experiments

\section{Titan laser enables new research}

Livermore scientists and engineers have developed a new intense shortpulse laser that will open a new world

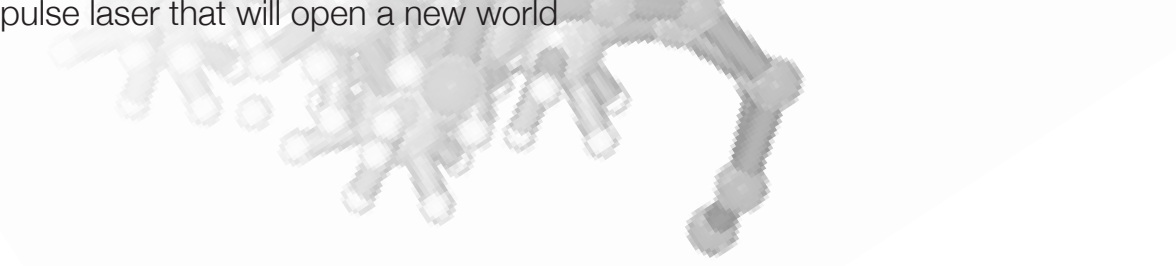


of research in high energy density physics. Funded by the Laboratory Science and Technology Office, the Titan laser is the product of a two-year collaboration between the Physics and Advanced Technologies, National Ignition Facility, Chemistry, Materials, and Life Sciences, and Engineering directorates at Livermore. When completed in 2006, Titan will be the Laboratory's first combined ultrashortpulse (sub-picosecond) and longpulse (nanosecond) laser operating at hundreds of joules of laser energy in each of its two beams. The short-pulse beam of Titan achieved first light in June 2005 and was officially dedicated for commissioning experiments in October 2005.

Titan is one of the few petawattclass lasers in the world. The shortpulse beam consists of a seed pulse generated with an optical parametric chirped pulse amplifier, which is amplified in the optical chain of the long-pulse Janus laser. The amplified chirped pulse is then compressed in time using a pair of very large, 40 centimeter $\times 80$ centimeter gratings in vacuum. The grating compressor - a key component of Titan-takes advantage of the novel, Livermore-developed multilayer dielectriccoated grating technology, which enables maximum laser intensity on target. The Titan short-pulse beam is capable of producing pulses of 400 femtosecond to 100 picosecond duration, with energy of 300 to 450 Joules, at a wavelength of 1053 nanometers. The long-pulse beam is the second arm of the Janus (Nd-glass) laser, which provides up to nanosecond pulses with 200-1000 Joules of energy.

The Titan laser beams are transported into the target chamber allowing different relative orientations of the two beams. The 3-meter diameter chamber is designed for a flexible arrangement of diagnostics enabling a variety of high energy density and high-field physics experiments. Initial experiments will include the production of high-energy proton beams and their use for isochoric heating of samples in materials studies, and for proton-radiograhy and deflectometry; the generation of intense, highenergy $\mathrm{K}$-alpha radiation for $\mathrm{x}$-ray radiography, imaging, and diffraction from laser-heated targets; and studies of the fast ignition concept for inertial confinement fusion.

\section{Contact: Andrew Ng (925) 423-4429 ng16@IInl.gov}

\section{High-speed images capture ripples in hot fusion plasma}

A team of scientists has opened a new window into the complex behavior that occurs at the edge of a 100-milliondegree fusion plasma. Using advanced high-speed cameras, the team obtained very detailed, three-dimensional images of plasma instabilities known as Edge Localized Modes (ELMs). Understanding the mechanism that lead to these instabilities will have important implications for the performance of the next generation of fusion devices, such as the International Thermonuclear Experimental Reactor (ITER), a major international project with significant U.S. participation.

The team, which included researchers from Lawrence Livermore, General Atomics, Oak Ridge National Laboratory, and the University of California, San Diego, performed the experiments at the DIII-D tokamak at General Atomics in La Jolla, California. The three-dimensional structure of the ELM was captured with the use of a high-speed camera operating with a very short (10-microsecond) exposure 
time to measure the blue light emitted by carbon ions as the ELM energy and particle pulse passed through the edge plasma. The images show that the ELM instability causes a helical distortion to the otherwise toroidally symmetric magnetic field. The spatial structure of the observed distortions is very similar to those calculated for the most unstable plasma modes localized near the plasma edge. This provides confidence that current theory can predict which instability leads to the ELM event. These results were reported at the annual meeting of the American Physical Society Division of Plasma Physics in October 2005.

Contact: Max Fenstermacher

(858) 455-4159

fenstermacher@IInl.gov

\section{Scientists find remarkable properties of lithium nitride}

Livermore researchers and their collaborators at the University of California at Davis and Argonne National Laboratory have discovered a new phase of lithium nitride that exhibits some remarkable properties. Lithium nitride is an interesting material because it is the only stable alkali metal nitride and is the most ionic of all known nitrides. It is a good ionic conductor with potential for use as an electrolyte in lithium batteries and as a hydrogen storage medium.

In experiments at the Advanced Photon Source at Argonne National Laboratory, the scientists compressed samples of lithium nitride over an extended pressure range up to 200 gigapascals, and probed its structural and electronic properties using a variety of $\mathrm{x}$-ray techniques. They found that, in the pressure range of 35-45 gigapascals, the material undergoes a phase transition to a cubic form, which exhibits increased structural and chemical bonding strength and becomes transparent due to a four fold increase in the electronic band gap. The cubic phase is uncommonly stable and quite compressible up to pressures of at least 200 gigapascals, making it a good candidate as an internal pressure standard in high-pressure experiments.

To explain the observed changes in structural and electronic properties, the researchers performed first-principles quantum mechanical calculations of the relevant phases of lithium nitride for various compressions. The results of the calculations for equilibrium volumes, bulk moduli, and transition pressure are all in good agreement with experimental values, and confirm the dramatic changes in properties upon transition to the cubic phase. The calculations also predict that cubic lithium nitride will remain an insulating solid up to pressures of at least 8,000 gigapascals, making it one of the most difficult materials to metallize. The results of this research appeared in the October 14, 2005, issue of Physical

\section{Review Letters.}

Contact: William Evans (925) 424-3356 wjevans@IInl.gov

\section{Simulating solidification of molten metals}

To evaluate the performance of nuclear weapons, scientists must understand how materials behave under extreme conditions. Of particular interest is pressure-induced solidification of molten metals. Because experiments at high pressures and temperatures are often difficult or impossible to conduct, scientists rely on computer models and simulations that have been validated with obtainable data. The simulations 
are performed on supercomputers sponsored by the Advanced Simulations and Computing (ASC) Program of DOE's National Nuclear Security Administration (NNSA).

In November 2005, a Livermore-IBM team received the 2005 Gordon Bell Prize for achieving a computing speed of more than 100 trillion floating-point operations per second (teraflops) while modeling the pressure-induced solidification of molten tantalum (see p. 16). This team produced the first atomic-scale model of solidification from the liquid phase, with results that were independent of system size used in the simulations. The record-setting calculation used Livermore's domain decomposition molecular-dynamics (ddcMD) code running on BlueGene/L, a supercomputer developed by IBM in partnership with the ASC Program. BlueGene/L at Livermore is currently the world's fastest supercomputer, having demonstrated 280.6 teraflops on the Linpack benchmark, which is the industry standard used to measure computing speed.

For the molten tantalum studies, the scientists simulated systems ranging from 64,000 to 128 million atoms compressed to pressure of 250 gigapascals at temperature of 5,000 kelvins. By varying the size of a simulation, the researchers gained confidence that their physics results were not affected by the size of the system being modeled. The 64,000-atom calculation showed two large grains, with a grain boundary spanning the simulation cell-an unrealistic result that indicated the system size was too small. In the simulation with more than 2 million atoms, the distribution of grain sizes was much more realistic. When the model size reached 16 million atoms, grain formation and growth were completely independent of system size. These calculations are the first step toward modeling nucleation and growth during solidification in a manner that allows scientists to directly link material behavior at atomistic scales to those at micrometer scales and above.

\section{Contact: Fred Streitz (925) 423-3236 streitz1@IIn.gov}

\section{National Ignition Facility looks toward ignition}

The results of a series of experiments, published in the November 18, 2005, issue of Physical Review Letters, indicate that the National Ignition Facility (NIF) is on the right path to successfully achieving ignition. The experiments were conducted by a large team of researchers, including scientists from the Physics and Advanced Technologies Directorate, using the first four laser beams at NIF. The team investigated plasma conditions inside hohlraums, small cylindrical cavities that convert laser light to $x$ rays. In future ignition experiments, $x$ rays will cause the implosion of a tiny capsule inside the hohlraum to create fusion ignition.

The four-beam experiments measured radiation temperature histories and correlated those with the rate of plasma production inside the hohlraum. The latter is important because laser energy delivered late in a long pulse (more than several nanoseconds) is ineffective for achieving ignition if the hohlraum is already filled with plasma formed from the irradiated walls of the hohlraum. Advanced diagnostics measured the $x$ ray spectra and radiation temperatures 
inside the hohlraum for various sizes of hohlraums and different laser pulse lengths. Instruments also imaged the $x$ rays that were energetic enough to escape the hohlraum. The hot electron production inside the hohlraum was inferred from the measured $\mathrm{x}$-ray spectra of the electron bremsstrahlung emission. The experimental data gathered were then compared to the predictions of theoretical and detailed computational models, which proved to be remarkably accurate.

The $x$-ray images of 1.6-millimeterdiameter gold hohlraums heated by a 2-nanosecond, flat-top laser pulse revealed, as expected, negligible plasma filling during the heating pulse. The scaling of the measured radiation temperature with laser power and hohlraum size was also consistent with the results of previous experiments. However, when same-size hohlraums were irradiated with a longer, 6-nanosecond, flat-top laser pulse, plasma filling of the laser entrance hole of the hohlraum was observed starting at about 4 nanoseconds. At the same time, there was an increase in the $x$ ray emission from the hot, gold plasma at the laser entrance hole, indicating increased localized absorption of laser energy before the beams reach the back wall of the hohlraum. With the same 6-nanosecond laser pulse into larger, 2.4-millimeter-diameter hohlraums, the gold plasma emission again remains concentrated at the back wall, indicating that the density of the hohlraum-filling plasma is moderate and that the laser still propagates into the hohlraum until the end of the laser pulse.

These findings served to validate the modeling and radiation hydrodynamics codes that are used for calculating the performance of full-size NIF hohlraums designed to achieve the conditions necessary for implosions leading to ignition.

Contact: Otto Landen (925) 424-5581

landen1@IIn.gov or

Marilyn Schneider (925) 422-0725

schneider5@IInl.gov

\section{Rapid detection of animal \\ diseases}

Since 2001, the U.S. has improved its ability to prevent and respond to a terrorist attack, but the nation's agricultural enterprise is a potential target for future attack. American livestock are particularly vulnerable to diseases that are endemic in other parts of the world but have not appeared in the U.S. for many decades. A great concern is footand-mouth disease (FMD), a highly contagious viral disease that has not been seen in U.S. livestock since 1929. Outbreaks of this disease in Europe, Asia, and South America during the past decade have heightened awareness of the potential danger.

Livermore researchers are developing new diagnostics for rapid detection of key animal diseases under the sponsorship of the Department of Homeland Security, in partnership with the U.S. Department of Agriculture (USDA). In 2005, the team developed a multiplexed assay - one that simultaneously tests a single sample for all similar diseases - for footand-mouth disease virus and animal diseases that cause clinical signs that are indistinguishable from FMD. Multiplexing reduces labor and the cost of reagents, and significantly increases the speed and convenience of testing. The assay relies on nucleic acid sequences to detect and identify 
the target organisms. It screens for both DNA and RNA viruses at the same time and detects the seven major subtypes of FMD. The test looks for 17 target signatures, including two that indicate the presence of FMD. In all, the 17 signatures represent seven diseases, three of which are foreign and four that are endemic in the U.S. The test also incorporates four internal controls to increase confidence in the results.

Thirteen laboratories within the National Animal Health Laboratory Network and the Plum Island Animal Disease Center have conducted an inter-laboratory evaluation of the Livermore assay. Initially, each laboratory analyzed the same 200 clinical samples containing known domestic viruses that mimic FMD. Follow-on evaluation will consist of analyzing clinical samples received from the field using both the standard diagnostic tests and the Livermore-developed multiplexed assay, and comparing the results. Once validated and accepted by the USDA, the new assay will allow laboratories to quickly diagnose FMD and diseases that mimic its symptoms.

The team is also developing a rapid, scalable, high-throughput laboratory system capable of analyzing the large number of samples expected during a foreign animal disease outbreak. The high-throughput system will be piloted at the California Animal Health and Food Safety Laboratory at the University of California at Davis, and the Colorado State University Veterinary Diagnostic Laboratory in Fort Collins, starting in the spring of 2006.

\section{Contact: Ben Hindson (925) 423-8667 hindson1@llnl.gov}

Detection of multiple human respiratory viruses

The control of communicable diseases ultimately rests on the speed at which the outbreak can be detected and identified. The rapid identification of infectious disease is crucial before an outbreak escalates into a full-scale epidemic or global pandemic. With the specter of an influenza pandemic a present reality, a method of rapidly identifying sick people for treatment and sequestration would be invaluable for healthcare workers, who would be on the front lines of a national publichealth emergency.

Many respiratory pathogens, as well as biothreat agents, cause symptoms commonly associated with influenza (headache, fever, malaise, body aches, etc.). While there are rapid tests available to identify influenza, they often have sensitivities of about 70 percent; this means 30 percent of the cases go undetected. Where rapid tests are not available, pathogens must be screened with viral culture or immunofluorescence techniques, which can take highly trained technicians up to 10 days to return a result. This time delay could be disastrous with the release of a communicable bio-agent or a global pandemic.

Livermore researchers are developing a new diagnostic tool for the rapid detection and identification of human respiratory viruses under funding from the National Institutes of Health and the Laboratory Directed Research and Development Program. This assay simultaneously tests for viruses using only a nasal swab. The assay relies on nucleic acid sequences to detect and identify the target organisms. It screens for both DNA and RNA viruses at the same time and detects eight major viral types by looking for 14 target nucleic acid signatures. These signatures represent eight different viral infections: influenza $A$, influenza $B$, respiratory syncytial virus, parainfluenza 1 and 2 , and adenovirus B, C, and E. The test also incorporates four internal controls 
to increase confidence in the results. By combining tests for each virus (multiplexing), the labor and reagent cost per test is lowered compared to seperate individual tests.

In addition, the researchers have developed a prototype of a fully autonomous instrument that will perform these assays in clinical settings. Called FlulD $D_{x}$, this instrument will require only fluid from a nasal swab and no special training to operate. All reagents and reactions are onboard. The operator simply introduces a sample and pushes a button. Results of the viral identification are available in three hours. FlulD $D_{x}$ will be undergoing field testing at the University of California at Davis Medical Center during the 2006 influenza season.

\section{Contact: Jim Birch (925) 422-7105 birch1@IInl.gov}

\section{Scientists get precise measure of a basic theory}

Laboratory researchers have made a new measurement that is 10 times more precise than previous measurements to test quantum electrodynamics (QED) an extension of quantum mechanics. The scientists entered a new realm in the search for deviations from the predictions of QED by measuring the spectrum of light generated in the extreme electric fields surrounding the nucleus of uranium. Deviations would have far-reaching consequences for understanding the universe because it would indicate that QED is not a complete fundamental theory of nature.

The researchers tested the theory using Livermore's SuperEBIT, an electron-beam ion trap, to strip uranium of all but three electrons, forming a plasma of uranium ions. Using high-resolution spectrometers in the experiments, the researchers measured with unprecedented precision the wavelength of extreme ultraviolet light emitted by the threeelectron (lithium-like) uranium ions. The very high precision of the SuperEBIT measurements allowed the team to extract an experimental value for the QED effects in which the polarized vacuum and the self-energy interacted with each other and themselves. noninteracting manifestations of QED. This research was published in the December 2, 2005, issue of Physical

Review Letters and was highlighted in Physics News in 2005 as one of the top physics stories of 2005.

Contact: Peter Beiersdorfer (925) 423-3985 beiersdorfer@IInl.gov

\section{Enhanced adaptive optics for Gemini Telescope}

Lawrence Livermore has been selected as the lead institution to build the Extreme Adaptive Optics Coronagraphy (ExAOC) for the Gemini Telescope. Researchers from the University of California at Berkeley and Los Angeles, NASA's Jet Propulsion Laboratory, the American Museum of Natural History, the Hertzberg Institute in Canada, and several smaller institutes will also collaborate on this project.

An enhanced adaptive optics system will allow astronomers to minimize the blurring effects of Earth's atmosphere, so they can detect planets about 30 to 150 light years from the solar system. Adaptive optics systems use light from a relatively bright star to measure and then correct the atmospheric distortions by bouncing light off a deformable mirror. The ExAOC system will have 3,000 to 4,000 actuators to control the deformable mirror. These actuators will be made of etched silicon microelectromechanical systems instead of reflective glass, which is used on the adaptive optics system at the 
W. M. Keck Observatory. With the new actuators, researchers will be able to adjust the shape of the mirror by several micrometers with a precision of less than 1 nanometer and, thus, will be able to correct for atmospheric distortions ten times better than the Keck system. Such an improvement in resolution will allow them to detect distant planets and learn more about how solar systems formed.

The ExAOC system is funded primarily by the National Science Foundation through the Association of Universities for Research in Astronomy. First light is predicted near the end of 2009 .

Contact: Bruce Macintosh (925) 432-8129 bmac@IInl.gov Reprinted from December 2005 issue of Science \& Technology Review of the Lawrence Livermore National Laboratory.

\section{Observing light echos from ancient supernovae}

A collaboration, which included three astronomers from Livermore, has observed scattered-light echos from three ancient supernovae centuries after the explosions. Supernovae are exploding stars, which are the brightest astronomical objects in the universe. Using the measured distance and apparent proper motion of the scattered-light-emitting regions, the researchers were able to determine ages of 610 and 410 years for two of the supernovae remnants.

As part of the SuperMACHO microlensing survey, the collaboration has monitored the central portion of the Large Magellanic Cloud (LMC) every other night for three months each year over four years. These observations have produced a very large database of images, which are ideal for searching for astronomical objects with variable brightness. Using an automated imageprocessing pipeline, the researchers easily identified the well-known echo of the recent supernovae, SN 1987A. They also found a number of very faint structures that had high apparent proper motions with vector directions inconsistent with the 1987A echo.

For each structure, the researchers extrapolated the proper motion vectors backward in time, pointing to a welldefined position corresponding to the origin of the light echo. In one case, the origin was identical to the known position of SN 1987A. Three other echo origins corresponded to the positions of known, relatively young supernova remnants that have been classified as likely thermonuclear events (type la SN) based on their $\mathrm{x}$-ray emission spectra. These findings were published in the December 29, 2005, issue of Nature.

The collaboration included the Cerro Tololo Inter-American Observatory (CTIO) and the Gemini Telescope in Chile, Ohio State University, McMaster University, University of Washington, Pontifica Universidad Católica de Chile, Livermore, and Harvard University. The reported observations were carried out on the 4-m Blanco telescope at CTIO, under the auspices of the National Optical Astronomy Observatory. NOAO is operated by the Association of Universities for Research in Astronomy, under contract with the National Science Foundation. Livermore's participation in SuperMACHO was supported by the Laboratory Directed Research and Development Program.

Contact: Kem Cook (925) 423-4634 cook12@IInl.gov 


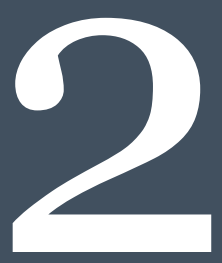

\section{People in the News}

Valuing scientific excellence,

leadership, and visibility 
The scientific and technological accomplishments of the staff in the Physics and Advanced Technologies Directorate are recognized outside the Laboratory through prizes, awards, and front-page publicity. Highlights in 2005 include:

Max Tabak, the

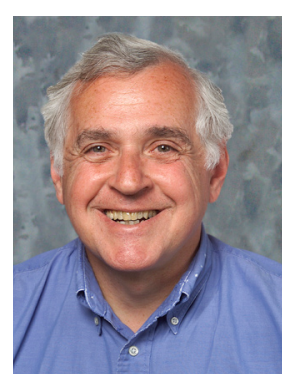
High Energy Density Physics Target Theory leader in the Fusion Energy Program, was one of two winners of the American Nuclear Society's

Edward Teller Medal for 2005. Tabak, a physicist in the Defense and Nuclear Technologies Directorate, received the award for his leadership of a Livermore research group that has made major contributions to a broad range of topics in inertial confinement fusion and high energy density physics, and has stimulated advanced research in plasma physics all over the world. He is best known as the lead inventor of 'fast ignition,' a technique that uses ultrapowerful, short-pulse lasers to directly ignite precompressed fusion fuel. His current research involves understanding how gigaampere currents of relativistic electrons propagate through matter. Controlling these power flows is key to the success of fast ignition and other applications of intense shortpulse lasers.

Two scientists in the Physics and Advanced Technologies (PAT) Directorate were elected Fellows of the American Physical Society (APS) in 2005.

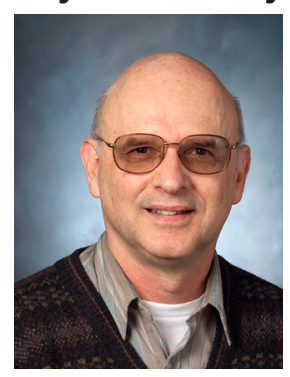

John Moriarty, a theory group leader in $\mathrm{H}$ Division, was recognized by the APS computational physics division for his "pioneering contributions to the first-principles quantum-based calculation of interatomic forces in $\mathrm{d}$ - and f-electron materials, with major impact on highpressure physics, multiscale modeling, and national security." Carlos Iglesias

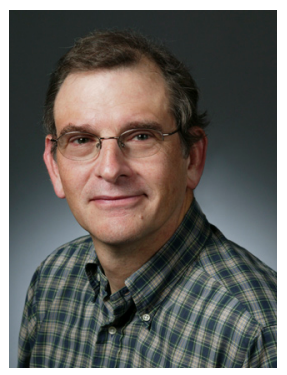
of $\mathrm{V}$ Division was cited by the APS plasma physics division for "ground-breaking contributions to the study of the production and transport of radiation in astrophysical and laboratory plasmas, including the development of the OPAL opacity code." The APS Fellowship recognizes those members who have made significant advances in knowledge through original research or who have made significant, innovative contributions in the application of physics to science and technology. Each year, no more than one-half of one percent of the current APS membership is elected to the status of Fellow. Currently there are 50 APS Fellows in PAT.

A team led by $\mathrm{H}$ Division physicist Fred Streitz was awarded the 2005 Gordon Bell Prize for pioneering materials science simulations conducted on the world's fastest supercomputerBlueGene/L. Other team members from Livermore included James Glosli in

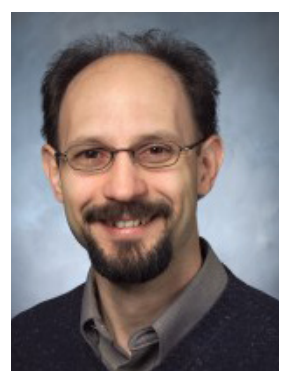

$\mathrm{H}$ Division, Mehul Patel in Defense and Nuclear Technologies, and Bor Chan, Robert Yates, and Bronis de Supinski in Computation. James Sexton and John Gunnels of IBM were also members of the team. Named for one of the founding fathers of supercomputing, the prestigious Gordon Bell Prize is awarded to innovators who advance high-performance computing. The researchers were recognized for "100+ Teraflops per second solidification simulations on BlueGene/L." Running a newly developed, three-dimensional molecular dynamics code (ddcMD) on 
BlueGene/L-the IBM machine that ranks first on the list of the world's Top500 supercomputers - the team investigated solidification in tantalum and uranium at extreme temperatures and pressures, with simulations ranging in size from 64,000 atoms to 524 million atoms.

Four teams of Livermore researchers won 2005 R\&D 100 Awards, known as the "Oscars of Invention." Each year, R\&D Magazine selects the 100 most technologically significant new products and processes, ones that are likely to produce the most benefits for the world at large. One of the awards involved significant contributions by scientists in the PAT Directorate:

Bioaerosol Mass Spectrometer (BAMS) is currently the only instrument that can detect and identify airborne spores of pathogenic organisms at low concentrations in less than one minute. BAMS can successfully distinguish between two related but different spore species. It can also sort out a single spore from thousands of other particles-biological and nonbiological - without false alarms. Previous systems had difficulty separating benign organisms from those that are pathogenic but very similar. PAT members of the award-winning team were James Birch, Matthias Frank, and Paul Steele of M Division. Eric Guard in the Chemistry, Materials, and Life Sciences Directorate has led the development of BAMS.

The BAMS team also received one of the two 2005 LLNL Science and Technology Awards. Established in 2000, these awards are given annually to recognize truly outstanding technical accomplishments across the Laboratory. PAT scientists sharing the award for BAMS included Michael Bogan, Matthias Frank, and Paul Steele of M Division and Norman Madden of I Division. The other award recognized key participants of the National Ignition Facility (NIF) Early Light Campaign for successful demonstration of the individual beam performance of the NIF laser and its utility to perform experiments. The NIF Early Light Campaign, concluded at the end of 2004, validated NIF as the premier high energy density science facility for doing quantitative experiments in four areas: laser-plasma interaction, hohlraum

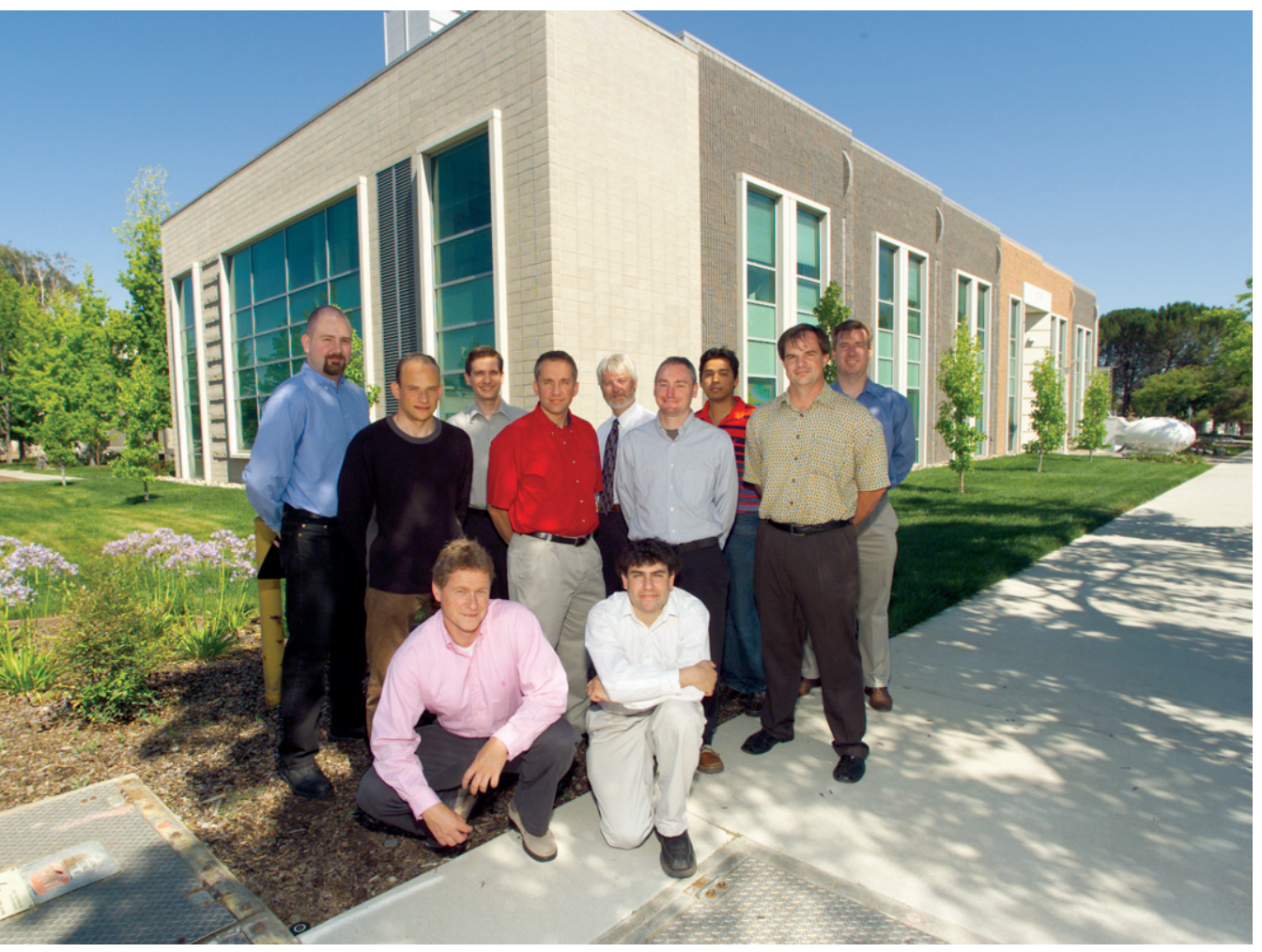

Members of the BAMS development team: (left to right, back row) Paul Steele, Todd Weisgraber, Bruce Woods, Abneesh Srivastava, and Keith Coffee; (middle row) Vincent Riot, Jim Birch, Herbert Tobias, and Eric Gard; (front row) Matthias Frank and David Fergenson. 
energetics, hydrodynamics, and shock physics. PAT members of the awardwinning team included Peter Celliers, Gilbert Collins, Joe Holder, Marilyn Schneider, and Bruce Young, all from $\checkmark$ Division; Laurent Divol of the Fusion Energy Program; and Franz Weber of $M$ Division.

The California Section of the American Physical Society (APS) has named a student award in recognition of Kennedy Reed, a physicist in V

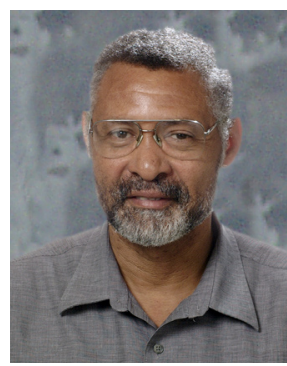
Division. During the 2005 fall meeting of the Section, the Kennedy Reed Award for Best Theoretical Research was given to three graduate students or postdoctoral researchers. The award consisted of three different cash prizes. Reed was recognized for his sustained educational outreach. For many years, he has directed the Research Collaborations Program for Historically Black Colleges and Universities and Minority Institutions within the Laboratory's University
Relations Program. He has also contributed significantly to the promotion of physics research and education in Africa, and has been a strong advocate for increased U.S. and international involvement with physics in Africa.

Reed was the recipient of the American Physical Society's John Wheatley Award in 2003.

After a national search,

\section{Ed Synakowski was appointed Fusion}

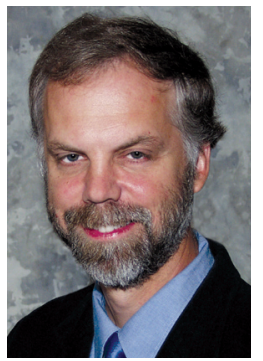
Energy Program Leader in the PAT Directorate. An internationally known plasma physicist, Synakowski was previously head of research at the National Spherical Torus Experiment at the Princeton Plasma Physics Laboratory. $\mathrm{He}$ is a previous recipient of the APS Award for Excellence in Plasma Physics, the Kaul Foundation Prize for Excellence in Plasma Physics, and the Donald Kerr Medal for Excellence in Physics. He is an APS Fellow and has published over 100 refereed journal articles.

\footnotetext{
Key participants of the National Ignition Facility (NIF) Early Light Campaign with then Laboratory Director Michael Anastasio and NIF Programs Associate

Director Ed

Moses (front row center), and Deputy Director for Science and

Technology Cherry Murray (front row right)
}

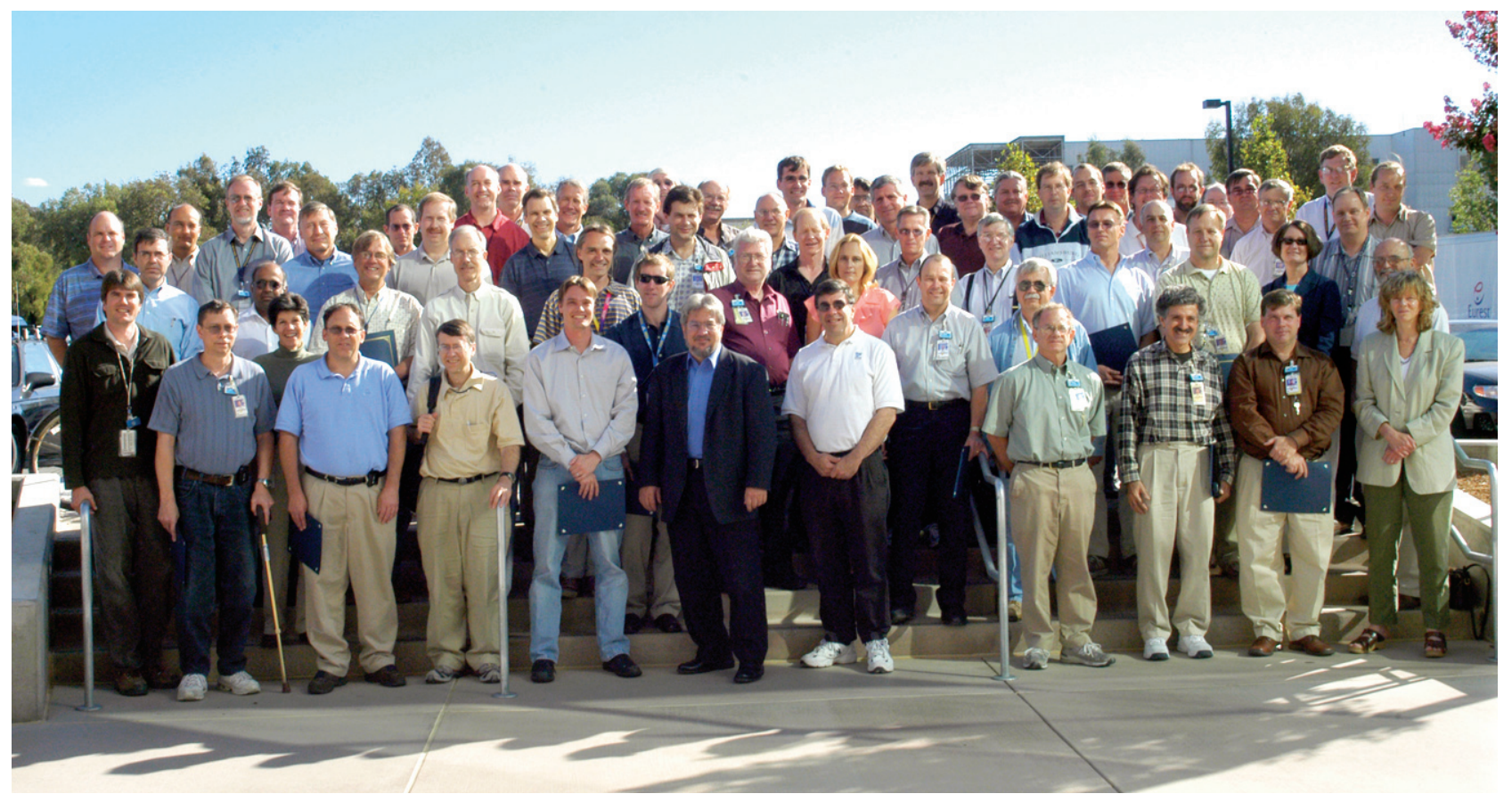




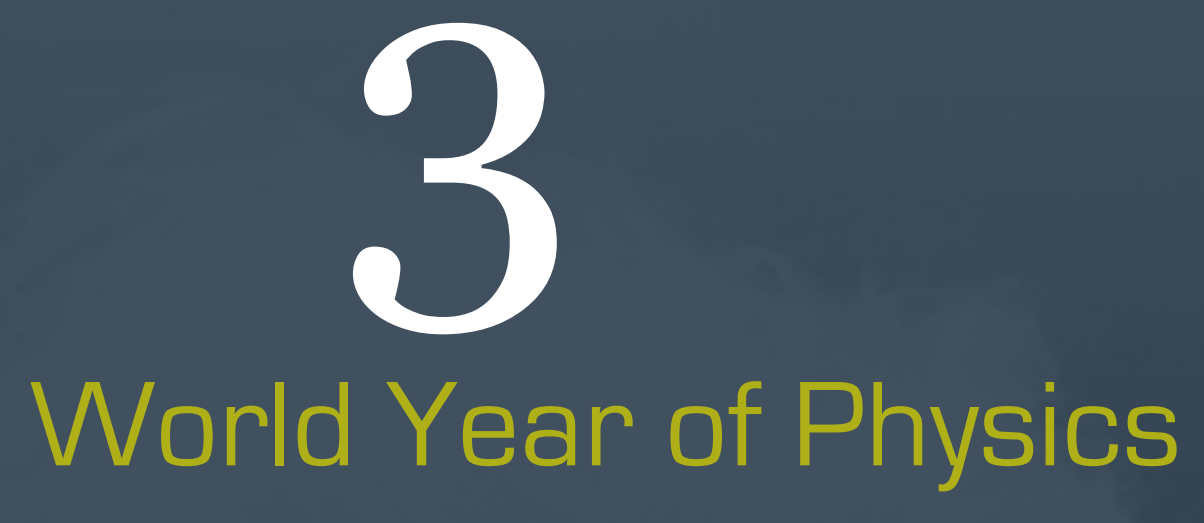

Building on Einstein's Legacy 


\section{Introduction}

In 1905, a 26-year-old Albert Einstein published four papers and a thesis that together revolutionized the field of physics and had far-reaching effects on technology and our understanding of the universe. To celebrate the 100th anniversary of Einstein's extraordinary accomplishments, the U.S. Congress, the United Nations, and various physics organizations declared 2005 the World Year of Physics. As part of Lawrence Livermore National Laboratory's participation in this yearlong celebration, Science and Technology Review-a monthly publication of the Laboratorypublished a four-part series, examining how Einstein's discoveries form the basis for much of the Laboratory's physics research (see www.llnl.gov/str/ May05/May05.html). Using excerpts from those four articles, we highlight ongoing research in the Physics and Advanced Technologies Directorate that would not be possible without Einstein's pioneering work.

\section{Applying Einstein's Theories of Relativity}

Einstein's most famous 1905 paper, "On the Electrodynamics of Moving Bodies," introduced the thenrevolutionary concept of relativity. Special and general relativity play an important role in our research into the physics of astronomical events such as gamma-ray bursts, black holes, and supernovae. In like manner, our supercomputer codes that model these events must account for relativity. Many of our astrophysics codes were adapted from versions developed originally for nuclear weapons research, which is based on concepts outlined in another Einstein paper. Finally, our work with machines that accelerate charged particles close to the speed of light would not be possible without incorporating the tenets of relativity.
Simulating the Big Bang. The change of mass and the apparent spatial length at relativistic velocities-close to the speed of light-are important factors in studies of matter in high-energy physics. For example, researchers are attempting to simulate the first microseconds after the big bang so they can explore new high-energy forms of matter and their origins. In a collaboration involving 40 institutions and 300 physicists from around the world, Livermore scientists are using the Relativistic Heavy-Ion Collider (RHIC) at Brookhaven National Laboratory to accelerate gold ions to within 99.995 percent of the speed of light. In RHIC, collisions at these extreme speeds cause the nuclei to shatter and transform into a hot plasma of their constituent quarks and gluons.

Scientists believe that quarks are the basic particles making up protons and neutrons. Additional particles called gluons mediate the strong force that holds quarks together and binds the protons and neutrons in atomic nuclei. Quarks and gluons possess a type of charge called color, which is the source of the powerful forces that bind the quarks together. Quantum chromodynamics - the theory of the strong interaction, or color forces - states that quarks and gluons can be liberated only under high-temperature conditions similar to those in the very early universe. At that time, in the first moments of the big bang, quarks and gluons are free as part of a quark-gluon plasma.

The collisions between two nuclei at $\mathrm{RHIC}$ are highly explosive, releasing more than a trillion electronvolts of energy in a volume the size of an atomic nucleus. This energy heats the nuclei to the high temperatures required for the formation of a quark-gluon plasma. At the high speeds attained at $\mathrm{RHIC}$, a special relativistic effect called Lorentz contraction is noticeable. The nuclei appear flat rather than spherical.

"Einstein's theory of special relativity is ingrained in the design of particle 
accelerators," says Livermore physicist Ron Soltz. "As the nuclei attain more energy, the velocity of the ions gets closer to the speed of light, but without ever reaching it. If we didn't have relativity, the way in which we use accelerators would be very different."

Soltz's team, which includes Livermore physicists Mike Heffner, Jennifer Klay, David Brown, and Ed Hartouni and postdoctoral researchers Jason Newby and Akitomo Enokizono, have been using the PHENIX particle detector at RHIC to study gold because the element has a heavy nucleus with 79 protons and roughly the same number of neutrons. Next, they plan to experiment with copper, which has a lighter nucleus with fewer nucleons. Soltz explains, "The heavier the nucleus of an element is, the more particles there are to participate in the interaction. Because of our success with gold-ongold collisions, we are trying a lighter element to determine if we will see similar plasma results."

The PHENIX collaboration published a comprehensive review of their experimental results in the August 8 , 2005, issue of Nuclear Physics A, which was devoted to the first three years of operations of $\mathrm{RHIC}$.

Codes to Model the Universe. In 1907 , Einstein began work on an extension to special relativity. Called the theory of general relativity, it described gravitation and its relation to the other forces of nature. With Einstein's theories of special and general relativity, researchers can model the forces in the universe to study its origins, determine how celestial objects influence one another, and then predict the evolution of these objects. Unfortunately, the equations of general relativity are complex and difficult to solve. Advances in computing methods and technologies over decades have been essential to understanding cosmological models, the universe, and astrophysical processes within them, by allowing scientists to solve the relativistic equations for realistic geometries on computers.

Laboratory physicists Dave Dearborn and Jim Wilson are collaborating with Grant Matthews from the University of Notre Dame to study how stars are destroyed by a black hole at the center of the galaxy. Their research indicates that stars known as white dwarfs become unstable when they are subjected to the strong gravitational field near a black hole. If a white dwarf passes too closely to a black hole at relativistic velocities, carbon and oxygen in the star's center are squeezed, initiating a runaway thermonuclear burn that blows the star apart. Because of the effect of general relativity, the central density required for nuclear ignition can be reached for stars with significantly smaller mass than that required for typical supernovae explosions. To better understand the conditions under which the central density can exceed the ignition threshold, the researchers have modeled the hydrodynamics of accelerating white dwarfs in the vicinity of black holes using a general relativity code developed previously by Wilson. They are using the Djehuty stellar evolution code developed by Dearborn's group to simulate in three dimensions the compression, thermonuclear burn, and explosion of the star. Preliminary results from these calculations appeared in the July 25, 2005, issue of Nuclear

\section{Physics A.}

\section{Observing Relativistic Effects Near} Black Holes. In collaboration with researchers at the Massachusetts Institute of Technology and the HarvardSmithsonian Center for Astrophysics, Laboratory astrophysicists Duane Liedahl and Christopher Mauche are developing computer codes to model the complex physics associated with $\mathrm{x}$-ray spectral emission from an accreting black hole. Although a black hole emits no light, its gravitational 
field can capture matter from nearby stars creating an accretion disk. Matter in the disk gradually spirals inward, converting gravitational potential energy into kinetic energy, thermal energy, and light. Because black holes have a small angular size, or observational angle, scientists cannot yet image these environments directly. Instead, they study the radiation emitted from a black hole's accretion disk, much of which emerges as $\mathrm{x}$ rays.

The collaboration developed the COMPASS code (computer models of the physics of accretion disk structure and spectra) in the 1990s. It can model the temperature, density, ionization state, and velocity of matter in the accretion disk. COMPASS incorporates HULLAC (Hebrew University and Lawrence Livermore atomic code), a code originally developed in the 1980s. HULLAC generates atomic models to determine the heating and cooling of the accretion disk and the spectrum of the emitted light. A Monte Carlo-based photon propagator has also been developed to track individual photons during their path through space-time. An early application of these codes to a black hole's accretion disk was published in the May 1, 2004, issue of the Astrophysical Journal.

Accretion disks are made up of common elements such as hydrogen, helium, oxygen, and iron. The ionized forms of these elements are the primary source of the characteristic $x$-ray radiation emitted from the disk. Normally, radiation emitted from plasmas appears as narrow lines on top of a continuum in the x-ray spectrum. However, for a black-hole accretion disk, the spectral lines are highly distorted by several classical and relativistic effects.

One such effect, called the Doppler effect, accounts for the change in the frequency of electromagnetic waves as an object moves toward or away from an observer. In an accretion disk, the Doppler effect broadens a narrow spectral line into a double-horned profile, which for the high velocities (150,000 kilometers per second or half the speed of light) near a black hole, extends from about 0.5 to 1.5 times the energy of the unperturbed line. Relativistic effects, such as time dilation and the beaming of the radiation in the direction of motion, further distort the line, dimming the red horn of the profile and brightening the blue horn. Finally, the emitted photons lose a significant fraction of their energy as they climb out of the gravitational well that surrounds a black hole, leading to a net red shift of the line profile. The resulting broad, skewed, and redshifted lines observed from Earth are the unmistakable signatures of black-hole accretion disks.

Liedahl and Mauche's computer models account for all these effects, allowing the team to calculate $x$-ray spectra as a function of black-hole spin and the angle of observation. These data are then compared to spectra collected from satellites such as NASA's Chandra X-Ray Observatory and the European Space Agency's XMM-Newton. X-ray satellites to be deployed in the next decade, such as NASA's Constellation-X Observatory, are expected to provide about 100 times the sensitivity of current satellites, allowing scientists to study in even more detail the extreme environments surrounding accreting black holes.

\section{Shedding Light on Quantum Physics}

In his first paper of 1905, "Concerning an Heuristic Point of View toward the Emission and Transmission of Light," Einstein explained some puzzling properties of light as a consequence of its particle-like nature. Einstein called light's discrete packets quanta; we now call them photons. He then used his insight into the particle-like nature of light to explain the photoelectric effect, in which light shone on a metal causes the ejection of electrons from the metal's 
surface. Later Einstein continued his inquiry into the properties of light and matter. In a paper published in 1917, "On the Quantum Theory of Radiation," he explained how radiation is absorbed and emitted by matter. In particular, he described three processes for how light interacts with atoms: spontaneous emission, absorption, and stimulated emission. These two papers are the foundation for our research in quantum physics, ionizing radiation, lasers, and advanced optical-imaging techniques.

Blackbody Radiation and Fusion. An ideal blackbody absorbs all radiation to which it is exposed and reflects none, so it appears black to the observer. The absorbed energy, however, is reemitted to maintain thermal equilibrium. It is the spectrum of this radiation-the distribution of intensity as a function of wavelength - that Einstein ultimately explained. In 1900, German physicist Max Planck calculated for the first time the observed distribution of radiation energy in blackbodies based on the assumption that the oscillating atoms in the walls of the blackbody do not emit radiation at all energies - only at highly prescribed values. Five years later, Einstein realized that Planck's explanation made perfect sense if the radiation in a blackbody was a collection of discrete light particles, which he called quanta.

Blackbody radiation is crucial for experiments performed at Livermore involving inertial confinement fusion, the process of compressing and heating hydrogen isotopes until they fuse together, releasing enormous amounts of energy. The goal of the National Ignition Facility (NIF) - Livermore's 192-beam, high-energy laser scheduled for completion in 2008-is to achieve fusion ignition and burn. The targets for the fusion experiments consist of a spherical fuel capsule containing deuterium and tritium, which is inside a hohlraum, a small cylindrical cavity (approximately 1 centimeter long and half a centimeter in diameter) usually made from gold. The laser beams are focused inside the hohlraum where they heat the inside walls creating black body radiation at a temperature of 3 million degrees. This radiation implodes the capsule, which compresses and ultimately ignites the fuel to initiate the fusion reaction.

The results of a series of experiments performed with the first four laser beams of NIF indicate that the facility is on the right path to successfully achieving ignition. The experiments, conducted by a large team of researchers including scientists from the Physics and Advanced Technologies Directorate, investigated plasma conditions inside hohlraums. The team measured radiation temperature histories and correlated those with the rate of plasma production inside the hohlraum. The results of these experiments, published in the November 18, 2005, issue

of Physical Review Letters, are summarized elsewhere in this report (see p. 10).

\section{Using the Photoelectric Effect to} Study Materials. Livermore scientists have been using the photoelectric effect to study the properties of solids as they rapidly evolve with time. Using a technique called $x$-ray photoelectron spectroscopy (XPS), a material is exposed to a short pulse of $x$ rays, and the energy distribution of the ejected electrons is measured. Every material has a unique electronic energy distribution. Studying these distributions - and how they change, for example, when the material is heated-offers scientists insights into the nature of the material. An intense, pulsed, mono-energetic $\mathrm{x}$-ray source is required. In 2001, Art Nelson and Jim Dunn began to use laboratory $\mathrm{x}$-ray lasers developed at Livermore to probe material properties in XPS experiments.

With funding from the Laboratory Directed Research and Development Program, Dunn's group developed a 
table-top soft $x$-ray laser source and combined it with advanced $x$-ray optics, to establish an $\mathrm{x}$-ray laser beamline for new science applications. The COMET (compact multi-pulse terawatt) $\mathrm{x}$-ray laser utilizes a short-pulse optical laser to heat thin foil targets to create the conditions required for amplified spontaneous emission of $x$ rays. The $x$-ray optics are fabricated from multi-layered materials designed to have the needed optical properties (e.g., reflectivity) at the wavelength of the $\mathrm{x}$-ray laser.

In the XPS experiments, the scientists use an optical laser pump and $x$-ray laser probe to understand the dynamics of a material on picosecond time scales. In this technique, the optical-laser pump quickly heats the material producing an excited state or causing a phase transition (e.g., melting). The x-ray laser measures the resultant perturbations by probing the state of the electrons in the material at various times. Because the pulse of an $\mathrm{x}$-ray laser is so short, one can get a snapshot by analyzing the kinetic energy distribution of the electrons ejected from the surface of the material.

In recent experiments, the researchers investigated the temporal evolution of the valence band electronic structure of thin copper foils heated with a very short (400 femtosecond) optical laser pulse. Variations observed in the photoelectron spectra with increased heating of the foils provided insights into the changing occupancy of the $3 d$ band of copper. Spectra of foils heated to about 15,000 degrees showed significant ionization due to ablation of foil by the laser heating pulse. The experiments also convincingly demonstrated the advantages of using an $\mathrm{x}$-ray laser probe for photoelectron spectroscopy: picosecond time resolution revealing transient effects and sufficient $x$-ray flux enabling "single-shot" experiments. These results were published in the October 10, 2005, issue of Applied Physics Letters.

\section{Random Walk through Time and Space}

Einstein's 1905 paper, "On the Movement of Small Particles Suspended in Stationary Liquids Required Molecular Kinetic Theory of Heat," has profoundly affected how scientists view the make-up of physical matter. In that paper, Einstein combined kinetic theory and classical thermodynamics to lay the theoretical foundation for observations confirming the existence and motion of atoms in fluids. Later that year, he extended the mathematical development of the theory to elucidate Brownian motion. By reconciling the laws of thermodynamics - the mechanical action of heat flow - with the kinetic theory of gases, he showed that the random motions of particles suspended in a fluid had to do with molecular movement and was affected by the temperature of the fluid. This work is the basis for much of the Laboratory's work in molecular dynamics, Monte Carlo statistical techniques, and physical chemistry.

Seeing Atoms. The scientists of Einstein's day had no hope of directly observing atoms, but a century later, new technologies are making that a reality. A team of researchers led by Livermore physicist Henry Chapman, under the auspices of the Laboratory Directed Research and Development Program, is developing a technique that will use the Linac Coherent Light Source at the Stanford Linear Accelerator Center to image complex biological molecules composed of 10,000 to 100,000 atoms. "We have moved in the space of a century from discussing whether atoms exist to actually being able to image atoms in crystals," says Chapman, "and we're on the threshold of imaging them in noncrystalline biological macromolecules, protein complexes, or viruses."

The basic technique for constructing these images is to inject a single molecule 
into the path of an incoming $x$-ray pulse and then measure the resulting diffraction pattern - the pattern of scattered $x$ rays just before the molecule explodes from energy absorbed. One image does not provide a complete three-dimensional picture of the molecule, so the process must be repeated many times. The scientists combine data from all of the images using a newly developed algorithm to build a three-dimensional model of the molecule, which then provides an average structure for that particular molecule.

Although the same type of molecule is being imaged each time, the diffraction patterns differ. "Brownian motion is partly to blame," says Chapman. "The molecules are jiggling and so are the atoms. Atoms also have thermal vibration, and even though they're bonded to each other, they're moving about. Some even swing at the end of their bonds, like a tether ball." To resolve this problem, the team is developing methods to align the molecules so they will always have the same orientation when imaged.

Recently a team comprising of researchers from Livermore, Lawrence Berkeley National Laboratory (LBNL), Arizona State University, and Stony Brook University has demonstrated the method of coherent $\mathrm{x}$-ray diffraction microscopy for imaging nonperiodic isolated objects at atomic-scale resolution. In experiments done at the Advanced Light Source at LBNL, the scientists obtained $x$-ray diffraction images of test objects with high resolution in all three dimensions, as determined by a quantitative analysis of the reconstructed volume images. These images were retrieved from the three-dimensional diffraction data using no a priori knowledge about the shape or composition of the object-a feat that has never been demonstrated for a nonperiodic object. These results were published in the May 2006 issue of the

Journal of the Optical Society of America, Part A.
From First Principles. Another group at Lawrence Livermore is simulating the motion of atoms from first principles using the laws of quantum mechanics as their only input. The Quantum Simulations Group investigates material behavior at high temperatures and pressures, at the nanoscale, and in solution. Two of these areas in particular involve Brownian motion: materials in extreme conditions and properties of fluids. Members of the group use firstprinciples molecular dynamics and quantum Monte Carlo codes to examine the behavior of atoms in liquids at the microscopic level. Their projects, many of which are funded by the Laboratory Directed Research and Development Program, involve quantum mechanical calculations for large numbers of atoms in a variety of physical conditions.

In recent work, the researchers, led by Giulia Galli (now a faculty member at the University of California at Davis), have investigated the behavior of liquid water and its structural, dynamic, and electronic properties at the molecular level. They have studied water not only at ambient conditions, but also under pressure, and in confined geometries, to determine how its structure and electronic properties change as thermodynamic conditions and geometry vary. It may be surprising that, at the beginning of the 21st century, scientists still do not completely understand-either from the theoretical or experimental perspective-the chemical bonding in this abundant material. At the nanoscale, determining the behavior of water and aqueous solutions is extraordinarily challenging. Experimental data are still sparse, and, in this regime, classical simulations can become unreliable. Quantum mechanical simulations, in contrast, require no experimental input, and, in principle, are capable of making accurate predictions. In practice, performing such simulations for very large numbers of atoms requires innovative algorithms and the speed of 
today's high-performance computers. Some of the group's research on water was published in the July 1,2005 , issue of the Journal of Chemical Physics.

Another example of the group's research involves simulations of liquid-solid interfaces, particularly the diffusion of water on surfaces. Recent calculations have shown that water does not uniformly wet the surface of silicon carbide, a leading candidate for biocompatible devices. Rather, water molecules prefer to bind along directions parallel to silicon atoms forming pairs (dimers) on the surface. The specific geometric arrangement of atoms on the outermost surface layer of silicon carbide is responsible for the orientation and coordination of water molecules, and, thus, plays a major role in determining surface reactivity. These results were published in the January 14, 2006, issue of the Journal of Chemical Physics.

\section{How One Equation Changed the World}

In his fourth 1905 paper, "Does the Inertia of a Body Depend upon Its Energy Content?," Einstein concluded that, as a consequence of special relativity, matter and energy are interchangeable. This work led to the famous equation $E=m c^{2}$. Thirty years later, physicists discovered fission and fusion, which demonstrated the conversion of mass into large amounts of energy. These discoveries made possible the nuclear weapons research done at Lawrence Livermore and Los Alamos national laboratories. It also paved the way for Livermore research efforts in peaceful nuclear power, such as magnetic fusion energy and inertial confinement fusion, as well as a variety of nuclear and particle physics experiments, in which matter and energy are interchanged.

Probing Subatomic Particles. The equation $\mathrm{E}=\mathrm{mc}^{2}$-together with the development of quantum mechanics and advances in nuclear physics - form the basis for particle acceleratorbased experiments in which physicists bombard targets with high-energy subatomic particles. Sometimes the particle collisions lead to new particles. In this respect, turning energy into matter is a well-tested method of uncovering the substructure of the universe. In one such project, Livermore physicists Peter Barnes, Doug Wright, and Ed Hartouni are participants in an international experiment centered at the Fermi National Accelerator Laboratory (Fermilab) in Illinois. The experiment focuses on measuring how one type of neutrino transforms into another type, a process called neutrino oscillation. The results promise to help scientists better understand particle physics as well as the role of neutrinos in the universe.

The Fermilab experiment, called the Main Injector Neutrino Oscillation Search (MINOS), uses a neutrino beamline, completed in early 2005 , that has an energy spectrum of 0.5 to 8 gigaelectronvolts. One goal of the MINOS experiment is to measure the rate at which neutrinos "change flavors," or oscillate from one type to another. The MINOS team uses two giant detectorsone at Fermilab and a 6,000-ton detector lying in a historic iron mine at Soudan, Minnesota. A narrow beam of neutrinos is generated and characterized by the near detector at Fermilab. The beam is aimed at the far detector in Minnesota. The neutrino beam energy is chosen so that the distance between the two detectors corresponds to an expected maximum in the probability that a neutrino produced at Fermilab will oscillate to another flavor. Comparing the muon neutrino beam flux and spectrum measured by the near detector with those from the far detector in Minnesota helps the scientist understand the properties of neutrino oscillations, and to determine the relative mass differences between the neutrino types.

Livermore physicists are also part of a project funded by the Laboratory 
Directed Research and Development (LDRD) Program to analyze data needed to accurately model the MINOS neutrino beam. The Fermilab Main Injector Particle Production (MIPP) experiment measures particle production from proton, pion, and kaon beams, ranging from 5 to 120 gigaelectronvolts, directed on targets including liquid hydrogen, beryllium, copper, carbon, bismuth, and depleted uranium. Pions and kaons are mesons, which help to bind the nucleus.

The Livermore scientists are particularly interested in measuring the species that are produced, their energies, and the angle at which they leave the target under bombardment with protons. These measurements aid the Laboratory's stockpile stewardship efforts. The researchers have been exploring the use of high-energy protons to create radiographs similar to $\mathrm{x}$-ray images. Proton radiographs could be used in stockpile stewardship to image deep inside explosive experiments and obtain information about materials too dense for $x$ rays to penetrate. However, proton radiographs tend to be blurry, in part because the proton beam that reaches the detector also contains subatomic particles produced as the beam passes through the object being imaged. Stockpile stewards need to know the exact identity of these secondary particles and how they affect the final image in order to make quantitative measurements.

\section{Two Roads to Nuclear Fusion.}

Controlled nuclear fusion could generate electricity without producing atmospheric pollution. Thermonuclear fusion of hydrogen is the energy source for the Sun and the stars. For such fusion reactions to proceed at high enough rates to be practical, the fusion fuel, which consists of isotopes of hydrogen (deuterium and tritium), must be heated to temperatures of about 100 million degrees Celsius. Two ways are being pursued to contain fusion fuel at the required temperature and density: magnetic confinement and inertial confinement.

The earliest controlled fusion effort at Livermore focused on magnetic confinement, in which the fusion fuel, in the form of an ionized gas or plasma, is trapped in a magnetic field for extended periods of time. The Laboratory's efforts directed toward magnetic fusion have evolved over the past fifty years. Today, researchers in the Fusion Energy Program collaborate with colleagues at General Atomics in La Jolla, California, to study the tokamak concept for fusion reactors. Experiments are conducted at the DIII-D machine at General Atomics to elucidate the complex behavior that occurs at the edge of the hot plasma confined in the tokamak. (See p. 8 for highlights of recent results.)

An alternative to the tokamak concept is Livermore's Sustained Spheromak Physics Experiment, built in 1997. In a spheromak, a self-organizing magnetized plasma forms naturally. The fluctuating magnetic fields and plasma flows create a dynamo that keeps the ionized hydrogen plasma alive and confined in space. Livermore's spheromak research is aimed primarily at increasing the plasma's temperature and gaining a better understanding of the turbulent magnetic fields and their role in sustaining the plasma.

(See the reprint of the article from the

September 2005 issue of Science and

\section{Technology Review.)}

Livermore researchers have also developed advanced computational models to simulate the properties and behavior of magnetically confined fusion plasmas, with a particular emphasis on the physics of the tokamak boundary region. The UEDGE edge transport code and the BOUT turbulence code are being applied to the interpretation of experimental data from DIII-D and other tokamaks. An example of such a simulation appeared in the March 1 , 2005, issue of the Journal of Nuclear

Materials. The new TEMPEST code, initially a four-dimensional neoclassical 
transport code, is undergoing validation against published experimental and theoretical results. TEMPEST is ultimately aimed at providing a complete description of the boundary region dynamics, including turbulence. Initial development of TEMPEST was funded by the Laboratory Directed Research and Development Program.

Results of these advances in computer simulations will aid the International Thermonuclear Experimental Reactor (ITER), which is the focus of a major international collaboration on magnetic fusion energy. The 10-meterdiameter ITER tokamak will be built in Cadarache, France, by a seven-party consortium including the European Union, Japan, Russia, U.S, China, India, and South Korea. Construction will begin in 2008, with the first plasma expected in 2016. When fully operational, ITER is expected to produce 500 megawatts of fusion power for 400 seconds. Contributions from the U.S. include diagnostics, superconducting central solenoid magnets, physics analysis, and tritium handling. Livermore has participated in the conceptual design of ITER and is contributing to the R\&D on central solenoid magnets, diagnostics and test blanket modules.

Using Lasers to Achieve Fusion Another way to achieve controlled nuclear fusion is to implode tiny capsules of frozen fusion fuel to the needed temperatures and densities using laser energy. This technique, called inertial confinement fusion, was pioneered at Livermore. At the high energy densities involved in this concept, the fusion reaction occurs in less than 100 trillionths of a second, and the inertia of the fuel itself is sufficient to provide the necessary confinement. The challenge of inertial fusion is that, to achieve energy gain, the laser must not only heat the fuel but also compress it to 1,000 or more times its initial density. The ignition targets being designed for the National Ignition Facility (NIF) will be compressed to a density and temperature about 10 times higher than those in the center of the Sun, in order to liberate more energy than is required to initiate the fusion reaction. Current plans call for the first fusion ignition experiments in 2010.

In 2004, Livermore researchers completed a series of experimental campaigns using the first four laser beams of NIF, as part of a program called NIF Early Light. Scientist from the Physics and Advanced Technologies Directorate played key roles in designing and fielding many of the diagnostic instruments used in these experiments. In addition, they were major participants in one of the campaigns, which investigated plasma conditions inside hohlraums, small cylindrical cavities that convert laser light to $x$ rays. In future ignition experiments, $x$ rays will cause the implosion of the tiny fuel capsule inside the hohlraum to create fusion ignition. (See p. 10 for more details.)

Einstein's work in 1905 was such an amazing burst of creativity that it has been called the annus mirabilis, or miraculous year. A measure of the lasting effects of Einstein's achievements is the way in which they still form the basis of work in pure and applied physics today. In that respect, this section of our report intended to show how Einstein's legacy is alive and well in the Physics and Advanced Technologies Directorate at Lawrence Livermore. We pride ourselves on forming teams of specialists to attack and solve challenging science and technology problems in the national interest. A hundred years ago, Einstein showed us how much one person can accomplish. 


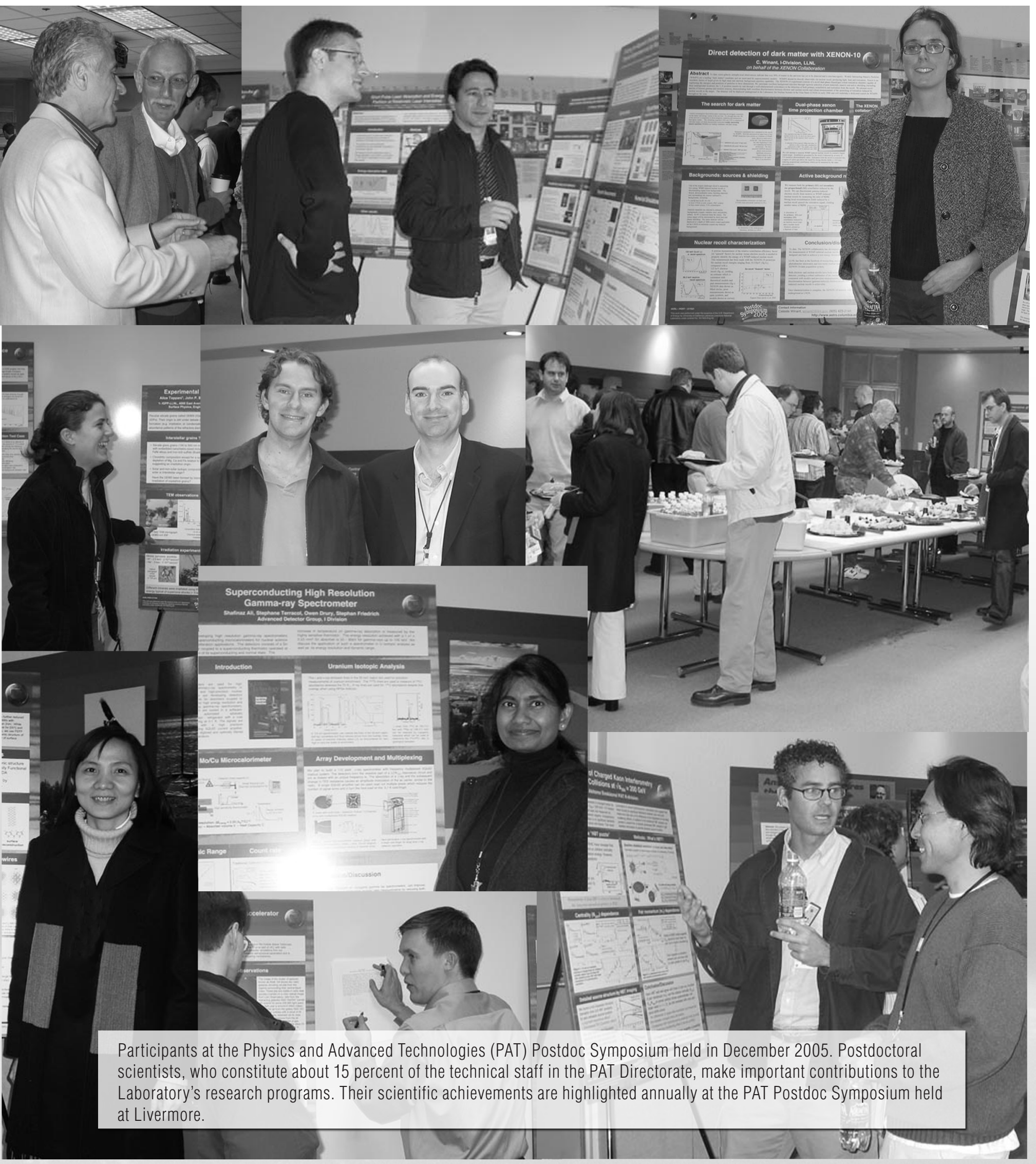


